\title{
Application prototype development, test and integration of shop-floor management applications
}

\author{
A. Martins(1),J. J. Pinto Ferreira(2), J. M. Mendonça(2) \\ (l)ISEP \\ Rua de S. Tomé, 4200 Porto, Portugal \\ (2)DEEC-FEUP/INESC-Porto \\ R. José Falcão 110, 4000 Porto, Portugal, Phone: 35122094300
}

\begin{abstract}
This paper describes a integrated set of tools supporting Prototype Development, Test and Integration of Shop-Floor Management Applications, as well as an illustrative case study. IncreasingRequirements advise that rapid prototyping of shop-floor software applications is fostered by the use of powerful modelling tools. The authors describe the use of a combined agent oriented/structured analysis approach to build the software system agent hierarchy and behaviour, which are further modelled as SDL process classes through a powerful Modelling Workbench. The result of this approach was the integration of two off-the-shelf tools, the Teamwork CASE Tool and the Modelling Workbench. This integration allows the SDL executable models to be used as emulated shop-floor applications. These application models, interacting with the prototypes of their user interfaces, as well as with the actual shop-floor simulation and production control models, may then be fully tested by the end-user in close-toreal conditions.
\end{abstract}

Keywords

Prototype development, shop-floor applications, modelling tools, SDL

\section{Shop-floor Software Application Prototype Development}

\section{Agent based analysis}

Special care must taken in the development of manufacturing management applications, specially those running at the shop-floor level, since application unreliability or inadequacy in meeting user requirements usually lead to expensive consequences. Within this context, software development approaches supported by prototyping and simulation may be used to forecast the application behavioue and thus prevent unexpected results. 
On the other hand, shop-floor applications, which belong to the broader group of distributed real-time applications, are hardly ever designed and implemented using common software tools and approaches. This is namely the case of SA-RT (System Analysis RealTime), which does not provide an adequate support to both model and code reutilization, and of most types of OOM (Object Oriented Modelling), whose models generally fail to describe clearly the functional behaviour of the system.

In order to deal with these problems, an agent based approach (Larvet, 1994) can be used where the best of two worlds is used: objects, modelling real world entities in the shop-floor and in the information system; and the structured decomposition of SA-RT, defining blocks/objects in terms of their functionalities, these agents interacting in a client/server environment. The system can therefore be modelled through an hierarchical top-down representation of interacting agents (using DFDs - Data Flow Diagrams) with increasing levels of detail. In parallel, a conventional object class hierarchy can also be used for agents' subcomponents reutilization, as figure 1 illustrates.

\section{Software Development Tools}

Following to the selection of the modelling paradigms, the need for tools capable of providing the adequate modelling support has emerged. The use of off-the-shelf software tools was set as a priority, as to allow a minimum use of development resources and ensure a scope of application as broad as possible. It was therefore decided to use a SA-RT CASE tool,

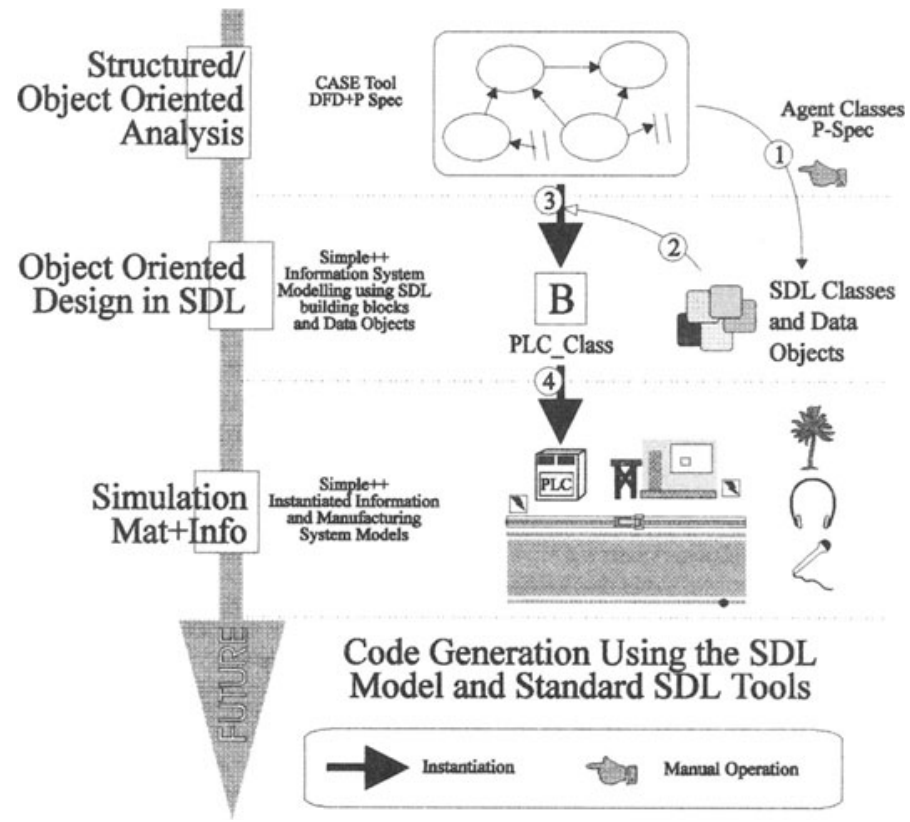

Figure 1 - The Software Model Life-Cycle 
Teamwork, for model structural representation and semantic validation. Model implementation and testing was achieved using Simple++ (AESOP, 1985), a state-of-the-art object oriented material flow simulation tool, which had been enhanced with a SDL-based information flow modelling tool. This integrated environment resulted in a Modelling Workbench (MW) supporting the modelling, construction and testing of shop-floor management applications (Pinto Ferreira, Pinto Ferreira, 1994).

\section{Tool Integration and Support to the Complete Software Model Life-Cycle}

Using the agent based approach, a complete system requirements analysis is first undertaken using Teamwork. The outcome of this analysis, is a set of DFDs and associated Data Dictionary and Process Specification (P-specs), wich build the system structured description. However, in this description, the bubbles in the DFDs represent agents, not processes as in SA-RT, whereas the data flows represent methods/services calls between agents (Martins, 1995).

Agent classes derived in the analysis are then fully modelled in the MW using SDL as the formal description technique (link 1 in figure 1). Some of these agent classes can be derived from existing classes in the library, as it is the case of the database agents in the Alarm Server example which will be presented later.

The final result of this phase is the system structured description built using the Teamwork CASE tool, and a set of executable SDL classes. Thes model can then be automatically built in the MW (link 3 in figure 1) by using the previously built SDL classes (link 2 in figure 1). Finally, the software model class can be instantiated in a particular model and executed/simulated, as illustrated in link 4 in figure 1.

Teamwork and Simple++ are however tools which were not developed to work together, so it was necessary to create a software module to read the descriptive model from Teamwork's database and transfer it to the Simple++ based Modelling Workbench. This was achieved by developing a program to generate a table representation of the system DFDs read from the Teamwork database, and to download it into a plain text file. This file is then processed by another program running in the MW, which automatically builds the actual resulting SDL software model (link 3 in figure 1).

The model is therefore built in the MW with the SDL system structure mapping the DFD based structure, where tasks/bubbles become SDL blocks and p-specs become either SDL processes or blocks (this will occur when a particular p-spec/agent is modelled as an entire SDL sub-system). All data stores are instanciated as SDL processes of a same class.

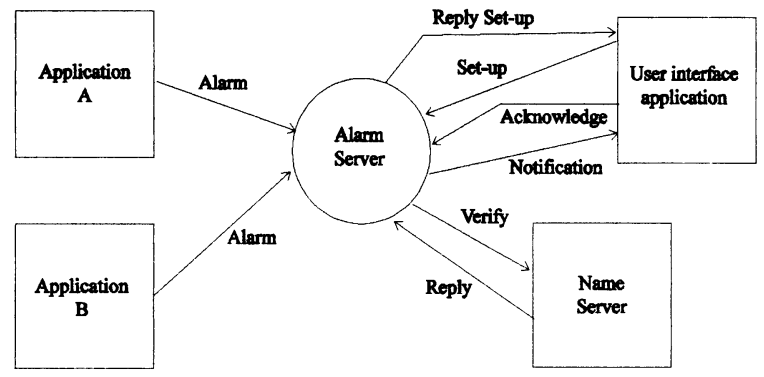

Figure 2 - Alarm Server Context Diagram 


\section{The Alarm Server, a practical example}

\section{System Analysis in the Teamwork}

Within the context of a manufacturing management system, the basic idea behind an alarm server is the use of a common alarm service as opposed to the use of one complex alarm handling module in each shop-floor application. The Alarm Server and its environment are presented in figure 2 through a context diagram. This diagram shows various applications interacting with the Alarm Server, namely two client applications using the alarm service (Applications A and B in figure 2), a user interface application which allows shop-floor operator notifications and alarm acknowledgement. A name server is used to check client application permission to set up the alarm server configuration database (set-up service in figure 2).

Figure 3 illustrates the partitioning of tasks of the alarm server. Three main agents are defined: the Alarm Handler, the Alarm Processor and the Database Manager. The first one receives the alarm and checks for its authenticity in the Records Table. If the alarm is valid, then it is sent to further processing in the Alarm Processor agent. A record of all alarms received is also kept in the Events Table.

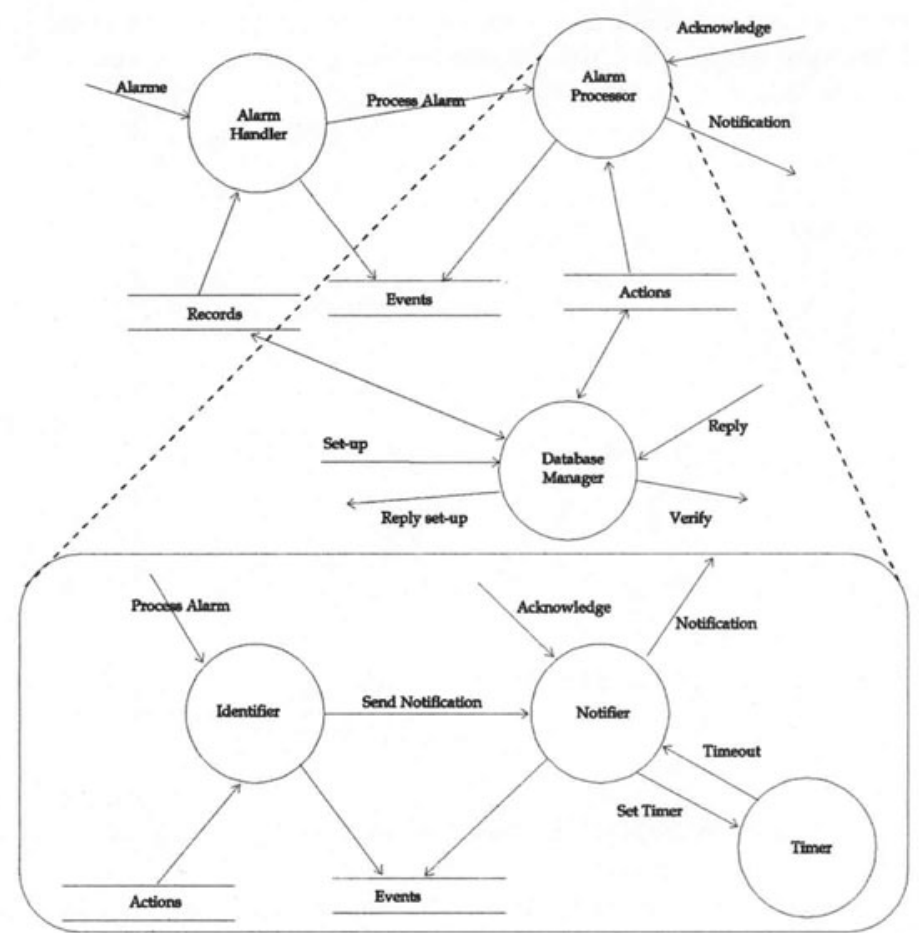

Figure 3 - Alarm Server Data Flow Diagram 
Within the Alarm Processor the Actions Table is used to decide the course of action to be followed whenever an alarm is received for processing. The information contained in this table is the alarm configuration, such as: the list of applications that should be notified upon the occurrence of the alarm, their addresses, pre-set alarm messages and acknowledge time-outs. This agent also receives confirmations from all notifications sent, updating the Event Table with the actual Notification Log. Figure 3 also portrays a DFD of the Alarm Processor agent, detailing its internal structure of agents.

\section{Loading the DFD Model into the Modelling WorkBench}

In this phase (link 1 in figure 1), each agent is modelled into two complementary ways: a SDL process, modelling the agent's behaviour; and a set of MW/Simple++ classes modelling agent's data objects. Building agent classes in the MW needs three steps:

- instantiation of a new process class,

- configuration of the process SDL state machine,

- building of data object classes to be handled by the agent.

Most of these classes can be derived from existing classes, considerably facilitating the programming effort and shortening the development time. As an example one could refer to agents modelling data stores, which only differ from one another in the format of the Database data object (figure 4).

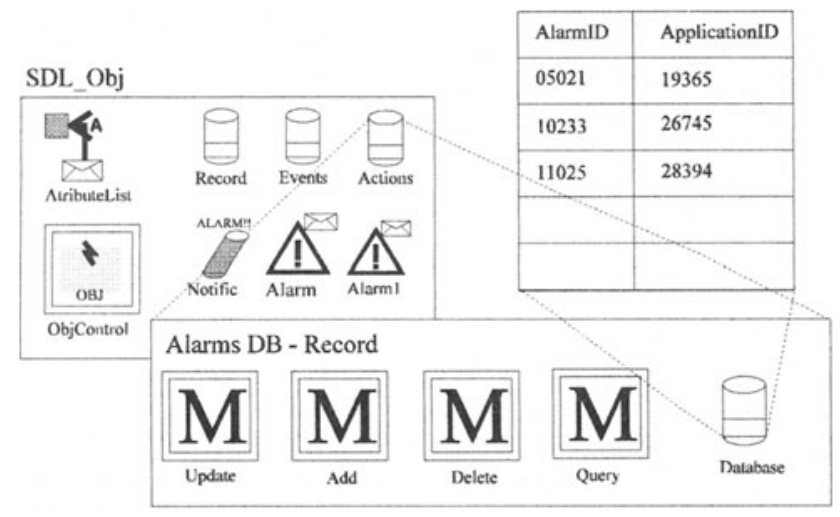

Figure 4 - Alarms DB Object, Table and Interface Methods

\section{Model Building}

As soon as all the classes needed are built (link 1 in figure 1), the building of the model is fully automatic, being done in three phases:

- generation of the model description into a text file, directly accessing Teamwork's database; - software class model bulding in Simple++, following the system's description in the previous file (links 2 and 3 in figure 1); 
- software model class instantiation in a particular model (link 4 in figure 1).

This last phase includes two stages:

- processes and data objects instantion and configuration;

- routing tables generation for simulation purposes.

The model is then ready for simulation to be executed.

\section{The Prototype Test and Validation Environment}

The paragraphs above described the development of a shop-floor software application prototype. The example provided was aimed at illustrating the general procedure to be followed during software development, the result being a SDL specification of that software module. The Alarm Server application was selected for its simplicity. This procedure may be nevertheless be followed in the development of any software module.

Once the software prototype development is complete, there is an obvious need for its test and validation. There is therefore the need to provide the user with the means to judge the new software module behaviour in the shop-floor environment. To fulfil this very important objective, one has considered that shop-floor exceptions should be first simulated in the laboratory environment, allowing the behaviour of the software module to be tested against a wide range of previous identified shop-floor conditions.

To accomplish this objective, the PROFIT ${ }^{(1)}$ messaging infrastructure was used as well as its modelling facilities provided by Simple++. Figure 5 illustrates this evaluation scenario where the following components cam be seen:

- The Message Transmission System (MTS). The MTS is a distributed socket-based mailbox mechanism, allowing software processes running in different machines to communicate across several UNIX, SCO-UNIX, DOS and Windows platforms.

- Three Simple++ processes (marked $\mathrm{S}++$ in the picture) exchanging messages through the distributed Message Transmission System and running a simulation of the actual Shop Floor environment, its simulated behaviour being embedded in the actual simulation model. Different shop-floor events may be modelled and used to simulate exceptional events, such as: machine failure, lack of raw material, quality problems, etc. The need for this Simple++ process only arises whenever the actual shop-floor is either not available or too expensive to run just for software test purposes. The production control executable model is used to monitor the shop-floor behaviour (emulated in the previous model). While the actual production is running in the shop-floor (i.e. its emulation), this production control executable model is being simulated in the real-time mode, so that the execution of the desired plan may be compared against the actual shop-floor execution. This model is normally used within the PROFIT environment, both for monitoring and control and for dispatching of work orders to the shop-floor. Moreover, this model is interacting with other shop-floor applications, such as the Alarm Server, as well as the Maintenance Management and Quality Management Servers.

${ }^{1}$ PROFIT (Production Optimisation through Flexible and Integrated Software Tools) Consortium: INESC-Porto (P); Tecnotron S.A. (P); IPA-FhG, Stuttgart (D); AESOP, Stuttgart (D) 
The SDL Alarm Server executable specification is simulating the actual Alarm Server. It is receiving events from the shop-floor, from the production control and from any other PROFIT Shop Floor Management application running in the system. On the other hand, it is sending alarm messages to the alarm client applications while requesting their acknowledgement whenever needed.

- An MS-Windows PC running an Alarm Client application. This PC is running the actual Alarm Client user interface prototype built using MS Visual Basic, MS-Excel, or any other user interface rapid prototyping tool.

\section{Running the Prototype in the Test and Validation Environment}

As illustrated, the integrated modelling and simulated environment provides the user with all the means to test and validate the desired software module. On the other hand, a quite flexible training environment may be built using the above described testing architecture. In this simulated environment, the system end-user may be confronted with many real life situations ranging from the very common to the most unlikely ones.

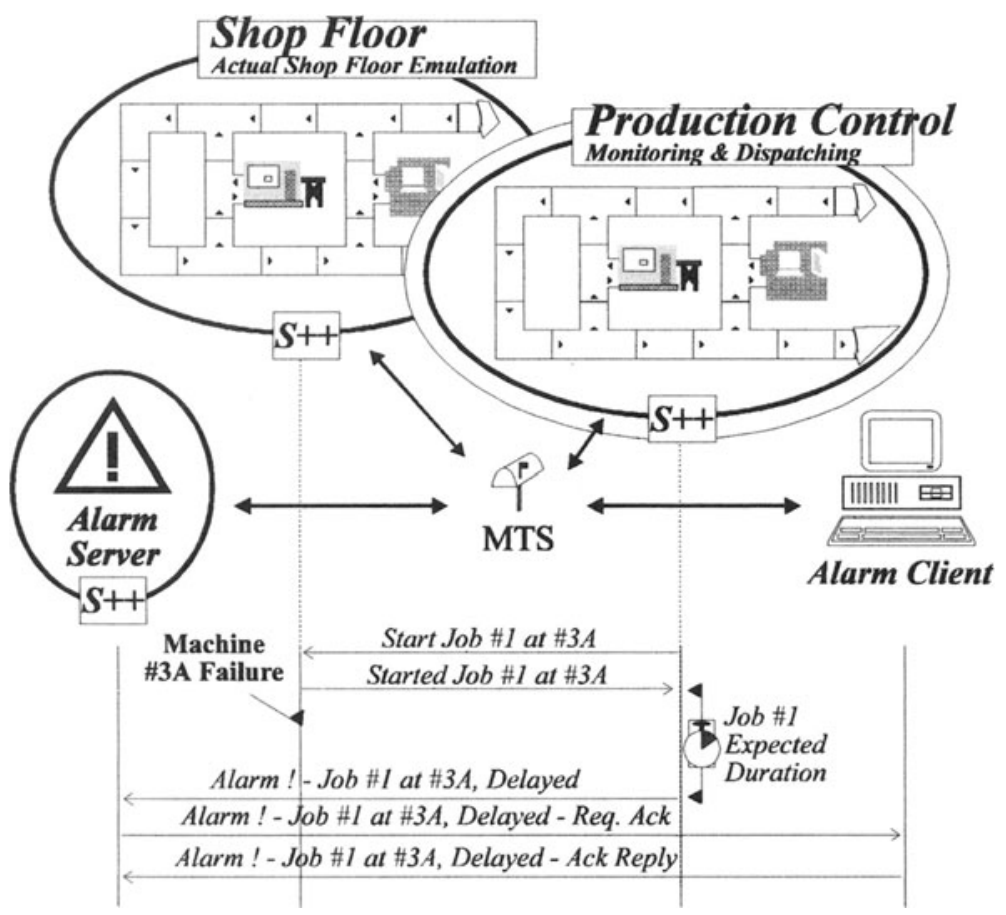

Figure 5 - Shop Floor Software Application Test \& Validation 
In this environment one is able to simulate, e.g. two different scenarios: (1) an operator issues an alarm from a terminal in the shop-floor, which is sent directly to the Alarm Server, (2) the production control model monitor detects a plan execution delay and issues an alarm which is then sent to the Alarm Server. Figure 5 illustrates the second situation with an example of a shop-floor simulation run where a failure of machine \#3A occurs. As this message arrives in the Alarm Server, the SDL executable specification handles that alarm message, and requests an alarm acknowledge from the alarm client.

\section{Conclusions}

This paper has presented experience with Prototype Development, Test and Integration of Shop-Floor Management Applications, building on an illustrative case study. Experience shows that special care must taken in the development of shop-floor management software applications, as errors or application inadequacy have generally immediate expensive results.

To avoid these problems, rapid prototyping of software applications should be fostered by the use of powerful modelling tools. As described above, agent oriented analysis was combined with conventional structured analysis based on Data Flow Diagrams. This agent based analysis produces both the agent hierarchy and the lower level agents behaviour, which are further modelled as SDL process classes in the Modelling Workbench environment.

The result of this approach was the integration of two off-the-shelf tools, a CASE tool (Teamwork) and a Modelling Workbench (running on top of Simple ++ ). This integration allowed the SDL executable models, which are automatically built within the MW by importing the DFD description from the Teamwork database, to be used as full featured emulated applications. These applications, interacting with their user interface prototypes, as well as with the actual shop-floor simulation and production control models, may then be fully tested by the end-user in close-to-real conditions.

\section{References}

J. J. Pinto Ferreira, J. M. Mendonça, Integrating Information and Material Flow Executable Models, An SDL-based Information Flow Modelling Tool, to be published in the International Journal of Computer Integrated Mamufacturing

J. J. Pinto Ferreira, J. M. Mendonça (1994) Integrated Modelling Tools for Shop Floor CIM Systems, IMSE'94-European Workshop on Integrated Manufacturing Systems Engineering, Grenoble

Philippe Larvet (1994) Analyse des systèmes: de l'approche fonctionelle à l'approche object, InterÉditions, Paris

A. Martins, J.J. Pinto Ferreira, J.M. Mendonça (1995) Da Análise e Especificação Estruturadas

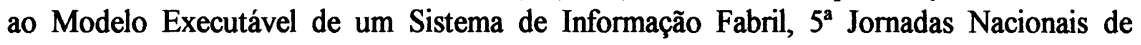
Projecto, Planeamento e Produção Assistidos por Computador, Guimarães

AESOP (1995) Simple++ User Manual, AESOP GmbH, Konigstrase 82, 7000 Stuttgart 1 